\title{
Isolated internal carotid artery dissection in a long-distance runner
}

\author{
Krzysztof Wachal ${ }^{1}$, Paweł Koczewski², Marcin Gabriel ${ }^{1}$, Wojciech Kociemba ${ }^{3}$ \\ ${ }^{1}$ First Department and Clinic of General and Vascular Surgery, Poznan University of Medical Sciences, Przemienienia Pańskiego Clinical \\ Hospital, Poznan, Poland \\ 2Department of Paediatric Orthopaedics and Traumatology, Poznan University of Medical Sciences, Wiktor Dega Orthopaedic Hospital, \\ Poznan, Poland \\ ${ }^{3}$ Department of Neuroradiology, Poznan University of Medical Sciences, Poznan, Poland
}

Videosurgery Miniinv 2016; 11 (4): 304-308 DOI: https://doi.org/10.5114/wiitm.2016.64449

\begin{abstract}
We present a case of internal carotid artery dissection (ICAD) in the precranial segment, which caused binocular visual impairment in a 49-year-old man during a marathon race. The incident lasted $3 \mathrm{~h}$, after which the symptoms resolved. Imaging tests showed internal carotid artery dissection. No ophthalmologic changes were identified. After 6 weeks of ineffective non-invasive treatment the patient underwent vascular surgery - stent implantation into the damaged artery. Detailed diagnostic tests and adequate treatment allowed us to achieve a good clinical result. Upon the suspicion of ICAD it is recommended to extend standard Doppler ultrasound performed with a linear transducer and evaluate the proximal segment of the internal carotid artery with a curved transducer as well.
\end{abstract}

Key words: long-distance running, marathon, binocular visual impairment, vascular angioplasty, carotid artery dissection.

\section{Introduction}

As the mechanism of carotid artery dissection is not entirely clear, it is assumed that it can be the effect of various, often minor traumas, or it can even be idiopathic. This raises the concern that a number of physical activities may expose "healthy patients" to the risk of serious vascular complications. The most common health problems in long-distance runners are lower extremity injuries, overload changes of the motor system and cardiopulmonary disorders. A number of incidents is noted during increasingly popular running events involving amateurs. They undertake intensive physical activities (marathon) feeling perfectly healthy but without prior diagnostic or function tests. The internal carotid artery dissection is very rarely diagnosed in long-distance runners. At the same time the symptoms may be scarce and limited to non-specific headaches or pain in the neck. One cannot exclude that a number of recorded sudden deaths in runners may be caused by this pathological condition. One can prevent these patients from incurring permanent damage and even save their lives thanks to extra attention when interpreting trivial symptoms, careful medical interview, quick imaging tests, correct diagnosis and adequate treatment.

\section{Case report}

A 49-year-old man, an amateur long-distance runner for 10 years, healthy and with no history of diseases, with positive results of periodic health examinations, had been suffering only from frequent headaches of unknown organic cause since early childhood. He had not suffered from any significant

\section{Address for correspondence}

Krzysztof Wachal MD, PhD, First Department and Clinic of General and Vascular Surgery, Poznan University of Medical Sciences,

Przemienienia Pańskiego Clinical Hospital, 1/2 Długa St, 61-848 Poznan, Poland, phone: +48 604 570 717, e-mail: krzywach@poczta.fm 
cardiac diseases apart from arrhythmia (single supraventricular extrasystoles) that did not require any medical treatment. Three months prior to the episode he had had his periodic exercise ECG, echocardiogram and Holter-ECG performed. None of them showed any contraindications for practising sport. Family history did not indicate any risk of vascular diseases either. After $3 \mathrm{~h}$ of the marathon race the patient started suffering binocular visual impairment in the form of flash lights variably narrowing his visual field. It was not accompanied by any other symptoms, neurological complaints or pain. The patient thought these symptoms were functional disorders caused by exhaustion and dehydration, so he only slowed down and finished the race. He did not suspect that this symptom might be caused by any trauma. After $2 \mathrm{~h}$ of rest the symptoms resolved spontaneously. After another $2 \mathrm{~h}$ the patient headed to the Accident and Emergency Department, where he underwent observation and was consulted by the neurologist, who did not recognize any abnormalities. He was also examined by the ophthalmologist and underwent ophthalmoscopy with the examination of acuity and field of vision. No abnormalities were found. He was discharged home with the diagnosis of function disorders caused by exhaustion. In another 7 days following the race the patient suffered only from permanent headaches, slightly more severe than the ones he used to complain about. Consequently he visited the neurologist again but again the examination did not reveal any abnormalities.

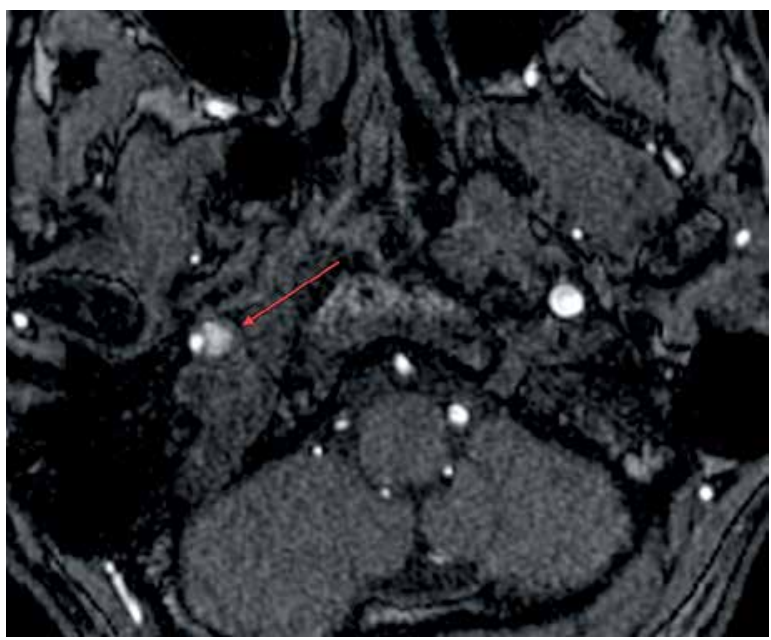

Photo 1. The magnetic resonance angiogram (MRA) showed the intramural thrombus in the right internal carotid artery
He was referred for cervical spine X-ray and carotid artery Doppler ultrasound. The headache resolved after 8 days. Cervical spine X-ray did not reveal any significant abnormalities and Doppler ultrasound showed normal morphology of carotid arteries with no blood flow disturbances. At the next neurological examination, having no symptoms (based on interview only) he was referred for a scheduled imaging test: magnetic resonance imaging (MRI) of the head. The MRI test was performed 3 weeks after the episode. The magnetic resonance angiogram (MRA) showed the difference in the diameter of internal carotid arteries in the intracranial segment with features of the reduction of influx into the right internal artery, and the patient was suspected to have an intramural thrombus in the right internal carotid artery (RICA) observed at the lower end of the image (Photo 1). Additionally, a small region of increased $\mathrm{T} 2$ and FLAIR signal was identified in the right parietal cortex suggestive of ischaemic focus. Three days later computed tomography angiography (angio-CT)

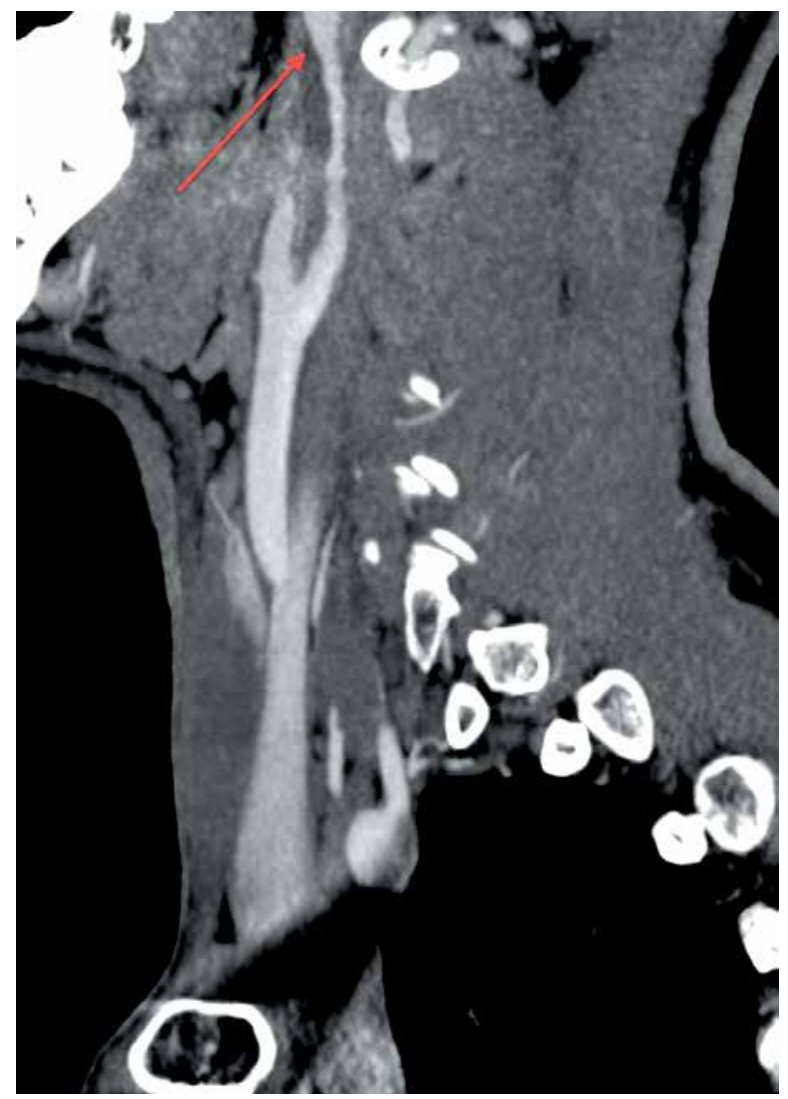

Photo 2. Angio-computed tomography - showing hypodense thrombi of the intra- and extracranial junction of the right internal carotid artery 
of the neck was performed. It showed right internal carotid dissection of $3 \mathrm{~cm}$ in the precranial segment, narrowing its lumen down to $1.6 \mathrm{~mm}$ (Photo 2). This diagnosis was confirmed by duplex Doppler examination, where the curved transducer showed the dissected part of the artery (Photo 3). Regardless of the presence of intramural haematoma and canal stenosis, a tear with normal blood flow was observed. The decision was made to start antiplatelet treatment with clopidogrel at the dose of $75 \mathrm{mg}$ (clopidogrel). After a week the antiplatelet therapy was replaced with anticoagulant treatment with rivaroxaban (rivaroxaban $10 \mathrm{mg}$ ) and a follow-up visit after 2 months was recommended. During therapy the patient gave up physical activity (he stopped his training but remained professionally active). After 6 weeks follow-up angio-CT of the neck, Doppler ultrasound of carotid arteries and Holter-ECG were performed on the hospital's neurology ward. Due to the lack of improvement in the arterial stenosis (without significant deterioration) and the ongoing blood flow in the dissected region the patient was referred for intravascular treatment. On the Neurosurgery and Neurotraumatology ward the patient underwent contrast-enhanced digital subtraction angiography (DSA) of the carotid and cerebral arteries, which showed the presence of a pseudoaneurysm of $17 \times 8 \mathrm{~mm}$ in the dissected region and critical internal carotid artery stenosis down to $0.5 \mathrm{~mm}$ (Photo 4). A stent of $7 \times 40 \mathrm{~mm}$ (Carotid Wallstent, Boston Scientific) was implanted into the internal carotid artery with a distal neuroprotection system (FilterWire EZ, Boston Scientific). The blood flow in the carotid artery reverted to normal with only trace flow in the region of dissection (Photo 5). In the postoperative period acetylsalicylic acid and clopidogrel were administered for 3 months, and after that acetylsalicylic acid alone. Due to moderate hypertension (135/90) the treatment was supplemented with perindopril arginine (perindopril $2.5 \mathrm{mg}$ )

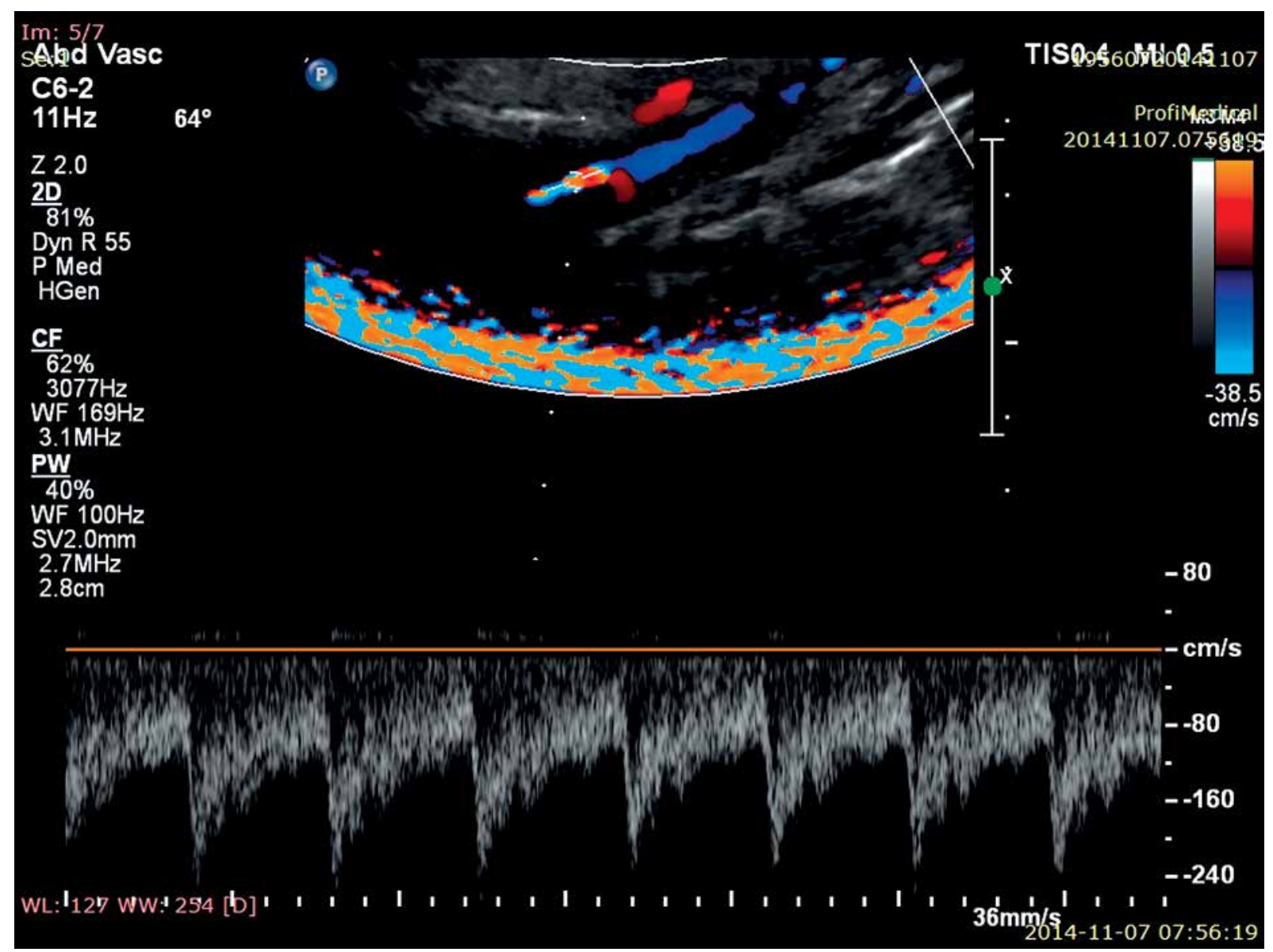

Photo 3. Internal carotid dissection in Doppler ultrasound with curved transducer 


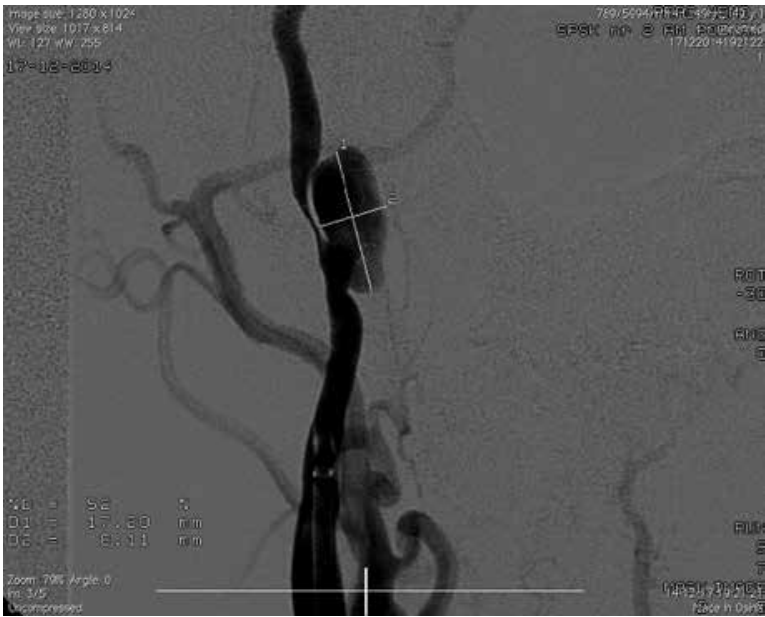

Photo 4. Digital subtraction angiography - presence of pseudoaneurysm of $17 \times 8 \mathrm{~mm}$ in the dissection region and critical stenosis of internal carotid artery down to $0.5 \mathrm{~mm}$

once daily. Follow-up Doppler ultrasound performed 1 month after the intervention showed correct blood flow through the artery of restored $5 \mathrm{~mm}$ diameter, without any intramural blood flow. Follow-up vascular MRI of the head involving the cranio-cervical junction was performed 3 months following the vascular procedure. It showed full remodelling of the vascular wall without intramural changes and with normal blood flow. Magnetic resonance imaging did not show the ischaemic lesion in the parietal lobe on the side of the dissection, visualized in the previous test. During a 6-month observation period the patient did not present any clinical symptoms, and radiological assessment confirmed almost complete resorption of the intramural thrombus. Complete diagnostic tests showed no disease which could predispose the patient to carotid artery dissection.

\section{Discussion}

Traumatic mechanisms of carotid artery dissection are usually the effect of blunt injuries to this region caused by car accidents. This pathology is also said to result from practising sports such a trampolining, diving, combat sports, skiing and snowboarding. Injuries are very rare in runners $[1,2]$. Medical practice shows dangers of rehabilitation procedures involving manipulation around the neck and perinatal traumas $[3,4]$. Twist of the neck with simultaneous compression of the arterial wall may lead to a tear of the intima, which leads to haemorrhage into the

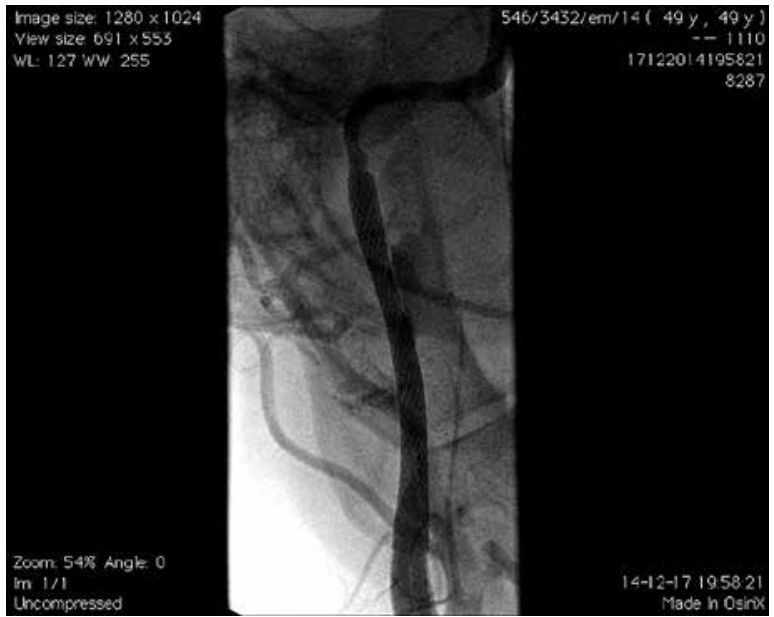

Photo 5. Digital subtraction angiography - after stent implantation into internal carotid artery

media [5]. The main mechanisms of the injury include hyperextension and twist or bend of the neck. It is important to assume that even minor trauma may cause internal carotid artery dissection (ICAD), as it is a recognized cause of ischaemic brain stroke in young people. Three retrospective studies which involved the review of 200 carotid artery dissections showed the average age of 45 years (in the range of 16-74 years). Episodes of central nervous system ischaemia occurred in 145 cases. In 22 cases transient ischaemic attacks were recognized. Five eye strokes, 2 cases of total loss of vision and 3 deaths were observed [6].

Very few published studies mention abnormalities connected with an elongated styloid process and its influence on the risk of internal carotid artery dissection [7].

The key therapeutic issue is the latent period, from the onset of dissection and ischaemia. In about $80 \%$ of ischaemic incidents symptoms appeared in the first 7 days, while in the remaining cases symptoms developed in the period up to 5 months. Therefore, it is recommended to administer antiplatelet medications for the period of 6 months following the recognition of such an incident [6].

The internal carotid artery dissection used to be considered a very rare incident. It was indicated by neurological and visual disturbances such as brain stroke or transient ischaemic attack (TIA) and Horner's syndrome $[4,8]$. However, as MRA and angio-CT were introduced, the number of recognized carotid and vertebral artery dissections started to grow. On the basis of 12 cases of carotid artery dis- 
section Stringaris et al. proved angio-MR to be more effective than traditional angiography [9]. In other publication Vertinsky et al. proved that computed angiograms had diagnostic efficacy comparable with MRA. Their studies involved 18 patients from 25 post-mortem examinations. The ultrasound technique, which is fast and uneventful, can also be a valuable diagnostic tool provided it involves using, apart from standard linear transducers, curved transducers to clearly visualize the whole extracranial segment of the internal carotid artery. It is also important to use transcranial transducers [10]. Zetterling et al. showed nearly $80 \%$ sensitivity in detection of carotid artery dissection. Early recognition and adequate referral of the patient are the key to therapeutic success [11]. Internal carotid artery dissection is a life-threatening condition and carries significant risk of stroke [12]. Our case shows that not only non-invasive treatment but also a surgical procedure helps to avoid serious complications. Non-surgical treatment is the treatment of choice and should last for a period of 3-6 months [8]. This case shows that internal carotid artery dissection may develop in young, fit and healthy people following great physical effort and without any visual injury. Numerous physical activities may pose a risk of ICAD, particularly marathon races. In this case it was not pain that was dominant, as one might expect. Therefore ICAD should be taken into account in differential diagnosis in all patients with sudden vision impairment after great physical effort. In most cases non-surgical treatment is sufficient to obtain recovery in the form of mural thrombus resorption. However, success is dependent on the complete coagulation of the dissected channel. In our patient a tear in the artery was recognized. Critical arterial stenosis posed a risk of expansion into distal and proximal regions and enlargement of the thrombus. The latter mechanism might have been responsible for the gradual shrinkage of the patent channel's diameter (from $1.6 \mathrm{~mm}$ to $0.5 \mathrm{~mm}$ ). A very detailed diagnostic procedure is the key to success [13]. On the basis of our case we believe that internal carotid artery imaging with curved transducers is a necessity in every patient with symptoms suggestive of microembolism, and in selected cases it should be supplemented with angio-MR or angio-CT. In such clinical cases standard imaging with a linear transducer is not sufficient, which was also observed in our patient. In case of blood flow in the dissected region, one should consider starting intravascular treatment earlier [14].

\section{Conflict of interest}

The authors declare no conflict of interest.

\section{References}

1. Lee WW, Jensen ER. Bilateral internal carotid artery dissection due to trivial trauma. J Emerg Med 2000; 41: 1935-41.

2. Nagumo K, Nakamori A, Kojima S. Spontaneous intracranial internal carotid artery dissection: 6 case reports and a review of 39 cases in the literature. Rinsho Shinkeigaku 2003; 43: 313-21.

3. Bell JS, Campbell DM, Graham WJ, et al. Do obstetric complications explain high caesarean section rates among women over 30? A retrospective analysis. BMJ 2001; 322: 894-5.

4. Demetriades AM, Miller NR, Garibaldi DC. Bilateral internal carotid artery dissection presenting as isolated unilateral Horner syndrome. Ophthal Plast Reconstr Surg 2009; 25: 485-6.

5. Fletcher J, Davies PT, Lewis T, Campbell MJ. Traumatic carotid and vertebral artery dissection in a professional jockey: a cautionary tale. Br J Sports Med 1995; 29: 143-4.

6. Baumgartner RW, Arnold M, Baumgartner I, et al. Carotid dissection with and without ischemic events: local symptoms and cerebral artery findings. Neurology 2001; 57: 827-32.

7. Zuber M, Meder JF, Mas JL. Carotid artery dissection due to elongated styloid process. Neurology 1999; 29: 143-4.

8. McCorry D, Bamford J. Painful Horner's syndrome caused by carotid dissection. Postgrad Med J 2004; 80: 164.

9. Stringaris K, Liberopoulos K, Giaka E, et al. Three-dimensional time-of-flight MR angiography and MR imaging versus conventional angiography in carotid artery dissections. Int Angiol 1996; 29: 143-4.

10. Vertinsky AT, Schwartz NE, Fischbein NJ, et al. Comparison of multidetector $\mathrm{CT}$ angiography and MR imaging of cervical artery dissection. AJNR Am J Neuroradiol 2008; 29: 1753-60.

11. Zetterling M, Carlstrom C, Konrad P. Internal carotid artery dissection. Acta Neurol Scand 2000; 101: 1-7.

12. Dziewas R, Konrad C, Dräger B, et al. Cervical artery dissection: clinical features, risk factors, therapy and outcome in $126 \mathrm{pa}$ tients. J Neurol 2003; 29: 143-4.

13. Gabriel M, Góźdź T, Zielonka D, Pawlaczyk K. Vertebral arterie dissection as a rare causa of central nervous system ischemia. Przegl Lek 2008; 65: 157-9.

14. Stanisić M, Winckiewicz M, Juszkat R, et al. Endovascular treatment of intramural hematoma of internal carotid artery after blunt trauma of neck inflicted with the seatbelt. VASA 2009; 38: 267-71.

Received: 17.03.2016, accepted: 15.11.2016. 